\title{
The Mediating Role of Self-compassion in the Correlation Between Perceived Social Support and Psychosomatic Symptoms Among Students with Gender as the Moderator
}

\author{
Zahra Feizollahi (iD) ${ }^{1}$, Hassan Asadzadeh (iD) ${ }^{2,}$, Saeed Bakhtiarpour (iD ${ }^{3}$ and Noorali Farrokhi (ii) ${ }^{4}$ \\ ${ }^{1}$ Department of Psychology, Science and Research Branch, Islamic Azad University, Tehran, Iran \\ ${ }^{2}$ Department of Educational Psychology, Faculty of Psychology and Education, Allameh Tabataba'i University, Tehran, Iran \\ ${ }^{3}$ Department of Psychology, Ahvaz Branch, Islamic Azad University, Ahvaz, Iran \\ ${ }^{4}$ Department of Assessment and Measurement, Faculty of Psychology and Education, Allameh Tabataba'i University, Tehran, Iran \\ "Corresponding author: Department of Educational Psychology, Faculty of Psychology and Education, Allameh Tabataba'i University, Tehran, Iran. Email: \\ asadzadehhsn@gmail.com
}

Received 2021 September 13; Revised 2021 November 09; Accepted 2021 November 28.

\begin{abstract}
Background: Psychosomatic symptoms manifest in different stages of human life. Students are vulnerable to the negative impact of these symptoms on their academic success and the possible consequences, such as illegal drug use and suicide attempts.

Objectives: The present study aimed to investigate the mediating role of self-compassion in the correlation between perceived social support and psychosomatic symptoms among university students.

Methods: This descriptive-correlational study was conducted on the students of the Islamic Azad University of Ahvaz in the academic year 2020 - 2021. The sample population included 372 students (178 males and 194 females) who were selected via simple random sampling. Data were collected using the medical outcomes study-social support survey, Neff's self-compassion scale (shortform), and DSM-5 somatic symptoms experiences questionnaire. Data analysis was performed using structural equation modeling. Results: A significant, negative correlation was observed between perceived social support and psychosomatic symptoms $(\beta=-0.14$; $\mathrm{P}=0.03)$, and a significant, positive correlation was observed between perceived social support and self-compassion $(\beta=0.34 ; \mathrm{P}=$ 0.001). Furthermore, a significant, negative correlation was denoted between self-compassion and psychosomatic symptoms $(\beta=$ $-0.52 ; \mathrm{P}=0.001)$. Self-compassion slightly mediated the correlation between perceived social support and psychosomatic symptoms ( $\beta=-0.19 ; \mathrm{P}=0.001)$, while gender did not moderate this mediating model.

Conclusions: According to the results, the modified model had a good fit. Therefore, self-compassion and perceived social support affected psychosomatic symptoms in the university students.
\end{abstract}

Keywords: Social Support, Empathy, Somatic Symptoms, Gender, Students

\section{Background}

Physical and psychological factors constantly affect human health. In recent decades, a new category of illness, known as psychosomatic diseases, has emerged, which is affected by emotional and psychological factors. Psychosomatic disorders encompass a wide spectrum of diseases, which are mainly characterized by physical signs and symptoms. These disorders indicate physical syndromes such as cardiovascular, respiratory, gastrointestinal, musculoskeletal, reproductive-urinary, and skin disorders, as well as migraine, vertigo, excessive fatigue, memory impairment, lack of concentration, dyspnea, nausea and vomiting, and insomnia; in these conditions, psychological complications are closely correlated with somatic symptoms (1). These disorders influence the interactions between the mind and the body, with the brain sending various signals via unidentified pathways that impact one's awareness, implying severe physical concerns (2).

Psychosomatic symptoms manifest in different stages of human life (3). Research suggests that approximately $50 \%$ of 14-year-old adolescents experience psychosomatic symptoms, and this rate may reach $75 \%$ at 24 years of age (4). Psychosomatic symptoms impact the behaviors, perceptions, and reactions of individuals to the surrounding environment. Students are highly vulnerable to the negative impact of these symptoms on their academic success and the possible consequences, such as illegal drug use and suicide attempts. Academic commitment, family/peer pressure, and other factors cause stress in students. As 
psychosomatic symptoms typically emerge in early adulthood, they could be identified and controlled in the first stages of the disease (5).

Perceived social support is another influential factor in the severity of psychosomatic symptoms. Social support refers to a sense of receiving attention from others, being valued by others, and a sense of belonging to a social network (6). According to sociologists, mental health and diseases are not merely biological and psychological facts, but also possess a social nature. Therefore, attention to the social factors that may affect health is of utmost importance, and the degree of perceived social support is considered a major influential factor in this regard. Nevertheless, few studies have elaborated on the mechanisms through which social relationships promote or endanger physical health. In the present study, we argued that selfcompassion may partially explain the correlation between perceived social support and somatic symptoms. In fact, self-compassion may mediate the correlation between social support and psychosomatic symptoms (7).

Self-compassion could mitigate anxiety-induced emotional distress, which often leads to psychosomatic symptoms (8). Emotions are among influential psychological factors that may cause or exacerbate psychosomatic diseases. The chronic effects of negative emotional inhibition increase sympathetic activity. Self-compassion is associated with coping styles and emotion regulation and may be regarded as an emotion regulation strategy operating variably. Notably, self-compassion does not inhibit the experience of undesirable emotions, but rather, attempts to accept these emotions. Promoting self-compassion could enhance positive emotions and reduce negative emotions (9).

Several studies support the theoretical framework encompassing the correlation between these variables. According to Grigaitytė and Söderberg (10), emotion selfregulation could mediate the correlation between social support and psychosomatic symptoms. Yeshua et al. (11) have also reported that high self-compassion moderates the effect of perfectionism on psychosomatic diseases. Moreover, Ferrari et al. (12) have stated that selfcompassion moderates the correlation between perfectionism and depression in adolescents and adults, while also mitigating the intensity of the correlation between maladjusted perfectionism and depression. According to Jeon et al. (13), self-compassion slightly moderates the correlation between social support and mental wellbeing, and the authors also confirm gender differences in an intergroup model comparison. Furthermore, Ling et al. (14) have reported that parental or peer support considerably influences the prediction of somatic symptoms and the psychological symptoms of depression in adoles- cents. Therefore, it could be inferred that low peer support (not parental support) in high school students acts as a mediator since it causes somatic and psychological depressive symptoms while dealing with frequent daily stressors (14). According to Yu et al. (15), self-compassion mediates the correlation between stress and psychosomatic diseases. Rajabi and Maghami (16) have also confirmed the mediating role of self-compassion in the correlation between various dimensions of attachment and mental wellbeing.

\section{Objectives}

The present study aimed to investigate the mediating role of self-compassion in the correlation between perceived social support and psychosomatic symptoms among university students in Ahvaz, Iran in 2020.

\section{Methods}

This descriptive-correlational study was conducted using structural equation modeling (SEM). The sample population included the students of the Islamic Azad University of Ahvaz in the academic year 2020 - 2021. To assess the proposed model, 400 students were initially selected via convenience sampling based on the number of the research variables. Due to the COVID-19 pandemic, this process was performed online. After explaining the research objectives, obtaining an online consent form, and ensuring the participants about the confidentiality of data, data collection was performed using questionnaires. After removing incomplete questionnaires and bivariate and multivariate outliers, the remaining 372 questionnaires were analyzed.

The inclusion criteria of the study were undergraduate students of the Islamic Azad University of Ahvaz Branch, no severe mental disorders, and consent to participate. The exclusion criteria were unwillingness to continue the study and failure to complete the questionnaires. In terms of ethical considerations, the participants provided informed consent, and the confidentiality of their information was ensured.

\subsection{Research Instruments}

\subsubsection{Medical Outcomes Study-social Support Survey}

The Medical Outcomes Study-social Support Survey (MOS-SSS), which was developed by Sherbourne and Stewart in 1991, was used to assess social support in this study. The survey consists of 19 statements and five subscales to measure the level of social support perceived by the respondents. MOS-SSS subscales include tangible support 
(material and behavioral help), emotional-informational support (positive emotions, empathy, and encouragement to express emotions), affectionate support (love and interest), and positive social interactions (the presence of people with whom recreational activities could be performed) (17). Togari and Yokoyama (18) have reported the Cronbach's alpha coefficient of 0.90 for this scale. In this study, the reliability of the MOS-SSS was confirmed at the Cronbach's alpha coefficient of 0.86 .

\subsubsection{Neffs Self-compassion Scale (Short-form)}

This 12-item scale is scored on a five-point Likert scale (Rarely $=1$, Almost Always $=5$ ). It measures three binary components in six subscales of kindness/self-judgment, common humanity/isolation, and mindfulness/overidentification. The short and long forms of the scale have a strong correlation $(r=0.97)$, and the test-retest reliability of the scale is reported to be 0.92 (19). In the present study, the Cronbach's alpha coefficient of the scale was estimated at 0.81 .

\subsubsection{DSM-5 Somatic Symptoms Experiences Questionnaire}

This 13-item scale encompasses four factors of health worries, experience of illness, problems in interacting with physicians, and consequences of illness with five, two, three, and three items, respectively. The items are scored based on a five-point Likert scale (Never $=1$, Always $=5$ ). The developers reported a mean Cronbach's alpha of 0.90 for the components (20). In the present study, the Cronbach's alpha coefficient was estimated 0.84 for the questionnaire.

\subsection{Statistical Analysis}

Data analysis was performed using path analysis to assess the proposed model in SPSS and AMOS version 23.0.

\section{Results}

The mean age of the male and female students was 21.12 \pm 1.43 and $20.73 \pm 1.84$ years, respectively. The students were divided into two groups based on their gender, including 178 males (48\%) and 194 females (52\%). Causal models are analyzed based on the correlation matrix. Table 1 shows the participants' descriptive indices (mean, SD, minimum and maximum score), as well as the results of Pearson's correlation-coefficient to evaluate the correlation between the predictive, mediating, and criterion variables. Accordingly, all the variables demonstrated significant correlations (Table 1). SEM was employed to simultaneously test the model of the hypothesized correlations. After the descriptive examination of the data, the statistical samples were divided into two groups based on their gender.
Prior to data analysis, the assumptions of multivariate normality, linearity, multicollinearity, and independence of errors were tested and confirmed. Skewness and kurtosis tests were also performed to examine the normality of data distribution. As the absolute magnitudes of the skewness and kurtosis coefficients were respectively $<3$ and $<10$ for all the variables, the assumption of the normality of data distribution was confirmed in both groups, as well as the entire sample population. To assess multicollinearity, the tolerance statistic and variance inflation factor (VIF) were used. For all the variables, the VIF was $<10$, and the tolerance statistic was $>0.1$, which justified the multicollinearity assumption. The assumption of the independence of errors was also tested using the DurbinWatson statistic, which was estimated at 2.05 for women and 1.79 for men; as this value was within the range of 1.5 2.5 , the assumption held.

In this study, three models were examined, including the general model, model of the female students, and model of the male students. Table 2 shows the fitness of the proposed and final models based on goodness-of-fit indices. Accordingly, the proposed model did not have a proper fit based on the root mean square error of approximation indicator (Table 2). Therefore, the model was modified by correlating the errors of the latent variables, and the results indicated the proper fit of the final model. Figure 1 depicts the standard path coefficients in the final model for the entire sample population.

Table 3 shows the structural model, paths, and standard coefficients of the final model for the entire sample population. Based on standard parameter coefficients and the corresponding significance level (Table 3), all the paths of the final model were significant. Therefore, a significant, negative correlation was observed between perceived social support and psychosomatic symptoms $(\beta=-0.14$; $\mathrm{P}$ $=0.03$ ). Moreover, perceived social support had a significant, positive correlation with self-compassion $(\beta=0.34 ; \mathrm{P}$ $=0.001$ ). The correlation between self-compassion and psychosomatic symptoms was also significant and negative $(\beta$ $=-0.52 ; \mathrm{P}=0.001$ ).

In the current research, percentile bootstrap and biascorrected bootstrap tests were performed to determine the significance of the indirect correlations between the variables and examine the mediating role of self-compassion (Table 4). To this end, the total effect of the predictive variable on the criterion variable was initially determined in the absence of the mediator. The obtained results indicated a significant correlation between perceived social support and psychosomatic symptoms without the mediator. In the indirect path and with the mediator variable, the correlation between perceived social support and psychosomatic symptoms was considered significant as 


\begin{tabular}{|c|c|c|c|c|c|c|}
\hline Variables & Mean \pm SD & Max. & Min. & 1 & 2 & 3 \\
\hline \multicolumn{7}{|l|}{ Female } \\
\hline 1-Perceived social support & $47.11 \pm 5.11$ & 37 & 52 & 1 & & \\
\hline 2-Self-compassion & $38.11 \pm 6.67$ & 58 & 111 & $0.36^{\mathrm{a}}$ & 1 & \\
\hline 3-Psychosomatic symptoms & $43.32 \pm 6.34$ & 21 & 58 & $-0.27^{\mathrm{a}}$ & $-0.54^{a}$ & 1 \\
\hline \multicolumn{7}{|l|}{ Male } \\
\hline 1-Perceived social support & $51.13 \pm 6.21$ & 42 & 55 & 1 & & \\
\hline 2-Self-compassion & $46.14 \pm 7.49$ & 69 & 113 & $0.32^{\mathrm{a}}$ & 1 & \\
\hline 3-Psychosomatic symptoms & $38.61 \pm 4.59$ & 23 & 55 & $-0.28^{a}$ & $-0.56^{a}$ & 1 \\
\hline \multicolumn{7}{|l|}{ Total } \\
\hline 1-Perceived social support & $49.55 \pm 6.09$ & 37 & 55 & 1 & & \\
\hline 2-Self-compassion & $41.95 \pm 7.42$ & 58 & 113 & $0.34^{\mathrm{a}}$ & 1 & \\
\hline 3-Psychosomatic symptoms & $51.06 \pm 5.75$ & 21 & 58 & $-0.27^{a}$ & $-0.55^{a}$ & 1 \\
\hline
\end{tabular}

Table 2. Final Model Fit Indicators

\begin{tabular}{lcccccccccc}
\hline Fit Indicators & $\chi^{\mathbf{2}}$ & df & $\left(\chi^{\mathbf{2}} / \mathbf{d f}\right)$ & IFI & TI & CFI & NFI & RMSEA & PCLOSE \\
\hline Initial model & 26.88 & 8 & 3.36 & 0.95 & 0.91 & 0.95 & 0.93 & 0.11 & 0.01 \\
Final model & 18.71 & 7 & 2.67 & 0.97 & 0.97 & 0.97 & 0.95 & 0.08 & 0.06 \\
\hline
\end{tabular}

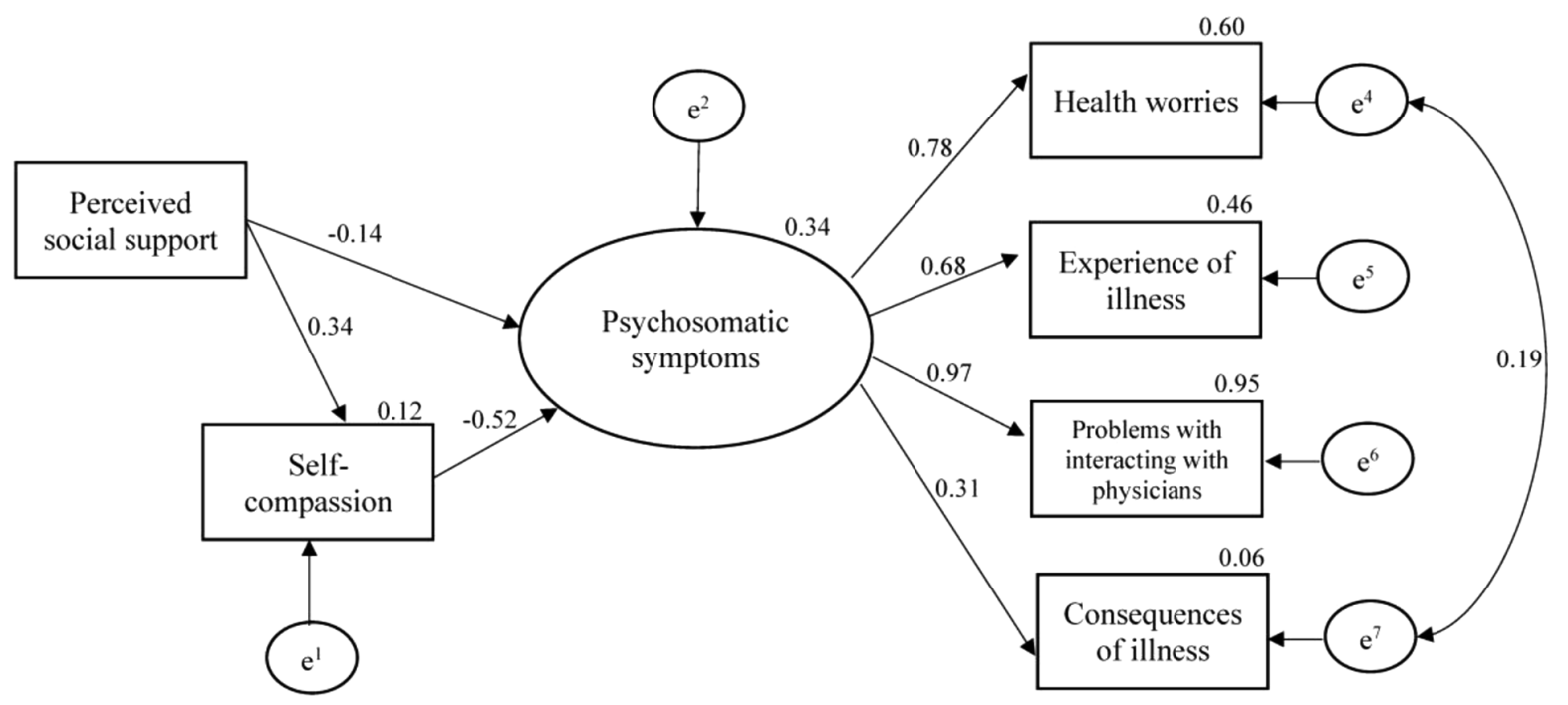

Figure 1. The standard path coefficients in the final model for the entire sample

well (Table 4). Therefore, it could be concluded that selfcompassion is partially affected by the impact of perceived social support on psychosomatic symptoms with a slightly mediating role in this regard.

In the present study, multigroup analysis was performed to compare the two models between the male and female students. The invariance of the measurement models in the female and male groups was evaluated by the comparison of the unrestricted model (model A) and the weight-restricted model (model B). Table 5 shows the unrestricted path coefficients of the male and female groups. Accordingly, all the paths were significant in both groups. 


$\begin{aligned} & \text { Table 3. Path Coefficients of Direct Effects Between Research Variables in the Final } \\
& \text { Model }\end{aligned}$
\begin{tabular}{lccc}
\hline Path & \multicolumn{3}{c}{ The Final Model } \\
\cline { 2 - 4 } & Path Type & $\beta$ & P \\
\hline $\begin{array}{l}\text { Perceived social support to } \\
\text { psychosomatic symptoms }\end{array}$ & Direct & -0.14 & 0.03 \\
$\begin{array}{l}\text { Self-compassion to psychosomatic } \\
\text { symptoms }\end{array}$ & Direct & -0.52 & 0.001 \\
$\begin{array}{l}\text { Perceived social support to } \\
\text { self-compassion }\end{array}$ & Direct & 0.34 & 0.001 \\
\hline
\end{tabular}

Figures 2 and 3 illustrate the unrestricted model (A) in both groups. Table 6 shows the comparison of the goodness-of-fit indicators of the unrestricted (A) and restricted (B) adjusted models in the two groups. Based on these indicators, the moderating role of gender was not confirmed. In other words, no difference was observed in any of the paths between the male and female students, and gender did not moderate this model.

\section{Discussion}

The present study aimed to investigate the mediating role of self-compassion in the correlation between perceived social support and psychosomatic symptoms among university students. According to the obtained results, perceived social support affects psychosomatic symptoms directly and indirectly through self-compassion. In other words, perceived social support exerted its effect on psychosomatic symptoms partially through self-compassion. Previous studies suggest a significant correlation between perceived social support and psychosomatic symptoms $(21,22)$. Accordingly, social support reduces psychological pressure and protects individuals against this issue. The knowledge and confidence of individuals in the fact that they could take refuge in their social network while dealing with mental pressure helps them evaluate life events as less threatening, which will eventually promote their resilience against the negative impact of stressors in a social context, thereby improving their physical and mental health. Social support improves health status by influencing stress outcomes; in other words, stressful periods in life urge individuals with less social support to demonstrate more symptoms of psychological disorders.

As no similar studies have been conducted in this regard, we could not compare our findings regarding the mediating role of self-compassion in the correlation between perceived social support and psychosomatic symptoms. Meanwhile, some studies have elaborated on the mediating role of self-compassion (12). As this mediating cor- relation constitutes two direct correlations, each correlation could be explained separately. Consistent with previous studies (23), a lack of perceived social support was observed to reduce the self-compassion of the students in the present study. Due to the lack of such support, individuals tend to view themselves as undesirable, thereby harboring negative emotions toward themselves and behaving toward themselves as others behave toward them. A sense of rejection may lead to undesirable outcomes such as selfattack, which make self-compassion difficult to attain. On the other hand, a high degree of perceived social support is associated with accurate emotion regulation. Therefore, self-compassion may develop in individuals with a high sense of social support as an emotion regulation and emotional balance strategy.

In the hypothesized mediating role of self-compassion in the correlation between perceived social support and psychosomatic symptoms in the current research, the second path was the correlation between self-compassion and psychosomatic symptoms. This finding is consistent with the research by Yeshua et al. (11). Individuals with a higher level of self-compassion are able to set health goals more effectively, strive to achieve these goals through adherence to treatments and health recommendations, and assess the path toward their goals. Self-compassion could affect students' general health since it influences emotional balance and results in a sense of self-care, awareness, and viewing one's deficiencies and failures without judgement (24). Self-compassion necessitates emotional expression, which is a process that stops the cycle of brooding, reduces loneliness, and enhances a sense of connection and relationship, all of which are associated with psychosomatic symptoms. By considering these two correlations simultaneously in the hypothesized model of the present study, perceived social support exerted an indirect effect on psychosomatic symptoms through self-compassion in addition to its direct effect; in fact, self-compassion slightly mediated this correlation.

According to our findings, gender did not moderate the general model. A moderating variable regulates the intensity and direction of the correlation between the independent variable (predictive) and the dependent variable (criterion). While no studies have examined the moderating role of gender, several studies have compared the mentioned variables between men and women, yielding different results. Currently, these symptoms are prevalent among students regardless of their gender (genderindependent). Moreover, sampling among the men and women of the same culture could justify the absence of the moderating effect of gender in the model of the present study. Owing to the undeniable advantages of social support, and given that humans are social by nature (espe- 


\begin{tabular}{|c|c|c|c|c|c|c|c|}
\hline \multirow{2}{*}{ Path } & & & & \multicolumn{2}{|c|}{ Indirect Effect } & \multicolumn{2}{|c|}{ Total Effect } \\
\hline & & & & $\beta$ & $\mathbf{P}$ & $\beta$ & $\mathbf{P}$ \\
\hline \multicolumn{4}{|c|}{ Perceived social support to psychosomatic symptoms through the mediating role of self-compassion } & -0.19 & 0.001 & -0.27 & 0.01 \\
\hline \multirow{2}{*}{ Path } & \multicolumn{2}{|c|}{ Female Students } & \multicolumn{4}{|c|}{ Male Students } & \\
\hline & $\beta$ & $\mathbf{P}$ & $\beta$ & & & $\mathbf{P}$ & \\
\hline $\begin{array}{l}\text { Perceived social support to psychosomatic } \\
\text { symptoms }\end{array}$ & -0.14 & 0.04 & -0.15 & & & 0.02 & \\
\hline Self-compassion to psychosomatic symptoms & -0.54 & 0.001 & -0.50 & & & 0.001 & \\
\hline Perceived social support to self-compassion & 0.35 & 0.001 & 0.33 & & & 0.001 & \\
\hline
\end{tabular}

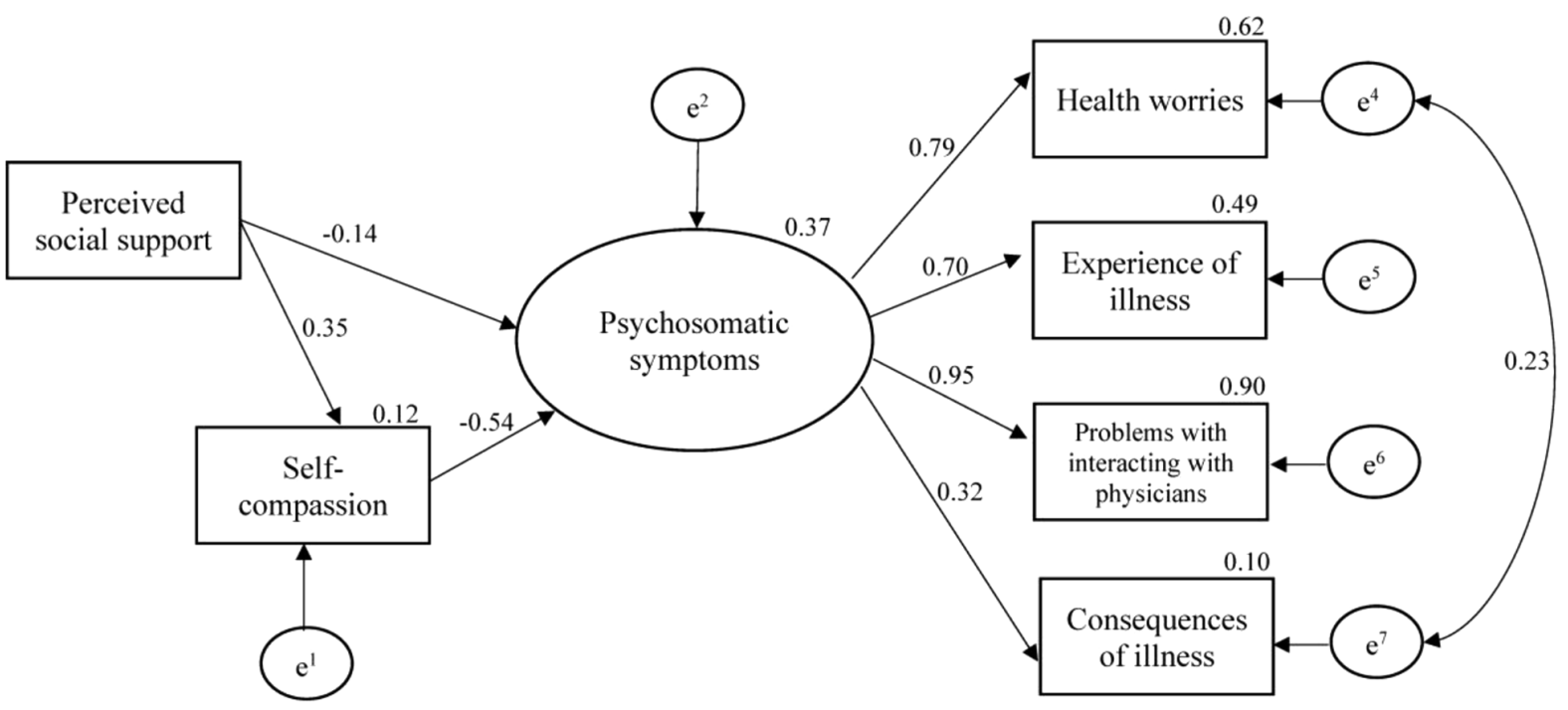

Figure 2. The standard path coefficients in the final model for the female students

Table 6. Comparison of the Goodness-of-Fit Indicators of the Unrestricted (A) and Restricted (B) Adjusted Models

\begin{tabular}{lcccccc}
\hline Fit Indicators & $\chi^{2}$ & df & GFI & CFI & RMSEA & $\Delta \chi^{2}$ \\
\hline Entire sample & 1.87 & 1 & 0.99 & 0.99 & 0.05 & 0.07 \\
$\begin{array}{l}\text { Unrestricted } \\
\text { model(A) }\end{array}$ & 41.25 & 16 & 0.97 & 0.96 & 0.06 & 0.06 \\
$\begin{array}{l}\text { Restricted } \\
\text { model (B) }\end{array}$ & 41.53 & 19 & 0.97 & 0.97 & \\
\hline
\end{tabular}

cially during adolescence and youth), providing students with training on self-compassion could mitigate their psychosomatic symptoms.

\subsection{Conclusions}

The findings of the current research should be interpreted and generalized while bearing its limitations in mind. This was a cross-sectional study, and the variables were measured at one time point. Moreover, the study was conducted during the COVID-19 pandemic, when the fear of illness and psychosomatic symptoms were exacerbated. Consequently, the correlations may assume different forms over time. Furthermore, the sample population of our study included the students of the Islamic Azad Uni- 


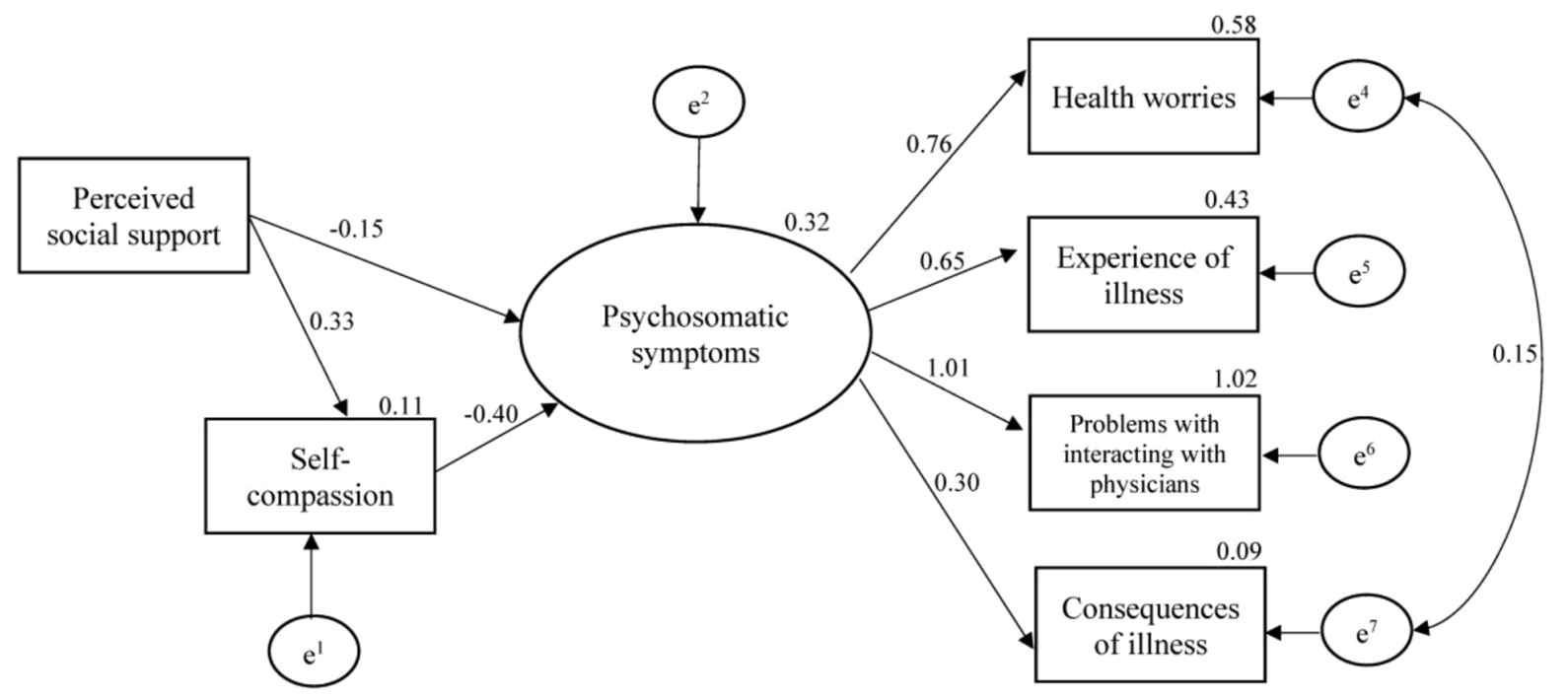

Figure 3. The standard path coefficients in the final model for the male students

versity of Ahvaz only, and the cultural and ethnic background of the region may have affected the results. Therefore, our findings should be generalized to other statistical populations with caution. Due to the limitations of correlational designs in explaining causal correlations, it is recommended that the effect of self-compassion on psychosomatic symptoms be examined in different populations through experimental studies by designing intervention packages and developing educational and interventional programs to promote social support and self-compassion. Complementary research with experimental and causalcomparative designs or studies with a panel data are also recommended.

\section{Footnotes}

Authors' Contribution: Zahra Feizollahi, and Hassan Asadzadeh developed the study concept and design. Zahra Feizollahi, and Saeed Bakhtiarpour acquired the data. Zahra Feizollahi, Hassan Asadzadeh, and Saeed Bakhtiarpour analyzed and interpreted the data, and wrote the first draft of the manuscript. All authors contributed to the intellectual content, manuscript editing and read and approved the final manuscript. Hassan Asadzadeh and Noorali Farrokhi provided administrative support.

Conflict of Interests: No conflict of interest to declare.

Ethical Approval: The study was approved by the Ethical Committee of Islamic Azad University-Science and Research Branch (code: 950459109$)$.
Funding/Support: This research did not receive any funding.

Informed Consent: Written informed consent was obtained from all participants.

\section{References}

1. Busch IM, Moretti F, Purgato M, Barbui C, Wu AW, Rimondini M. Psychological and Psychosomatic Symptoms of Second Victims of Adverse Events: a Systematic Review and Meta-Analysis. J Patient Saf. 2020;16(2):e61-74. doi: 10.1097/PTS.0000000000000589. [PubMed: 30921046]. [PubMed Central: PMC7386870].

2. Griffies WS. Impaired Mind-Body Connections in Psychosomatic Patients: A Contemporary Neuropsychodynamic Model. Psychodyn Psychiatry. 2019;47(3):317-42. doi: 10.1521/pdps.2019.47.3.317. [PubMed: 31448984].

3. Ozamiz-Etxebarria N, Idoiaga Mondragon N, Dosil Santamaria M, Picaza Gorrotxategi M. Psychological Symptoms During the Two Stages of Lockdown in Response to the COVID-19 Outbreak: An Investigation in a Sample of Citizens in Northern Spain. Front Psychol. 2020;11:1491. doi: 10.3389/fpsyg.2020.01491. [PubMed: 32625157]. [PubMed Central: PMC7314923].

4. Nawafleh HA, Al Hadid LA, Al Momani MM, Al Sayeh AM. The Prevalence of Psychosomatic Symptoms and Spirituality Levels among University Students in South Jordan. Health. 2018;10(1):107-21. doi: 10.4236/health.2018.101009.

5. Lindstrom M, Fridh M, Rosvall M. Economic stress in childhood and adulthood, and poor psychological health: three life course hypotheses. Psychiatry Res. 2014;215(2):386-93. doi: 10.1016/j.psychres.2013.11.018. [PubMed: 24332463].

6. Wang J, Mann F, Lloyd-Evans B, Ma R, Johnson S. Associations between loneliness and perceived social support and outcomes of mental health problems: a systematic review. BMC Psychiatry. 2018;18(1):156. doi: 10.1186/s12888-018-1736-5. [PubMed: 29843662]. [PubMed Central: PMC5975705]. 
7. Sumter SR, Baumgartner SE. Psychosomatic complaints in adolescence: Untangling the relationship between offline and online peer victimization, psychosomatic complaints and social support. Eur J Dev Psychol. 2016;14(4):399-415. doi: 10.1080/17405629.2016.1215980.

8. Neff KD, McGehee P. Self-compassion and Psychological Resilience Among Adolescents and Young Adults. Self Identity. 2010;9(3):225-40. doi: $10.1080 / 15298860902979307$.

9. Krieger T, Hermann H, Zimmermann J, grosse Holtforth M. Associations of self-compassion and global self-esteem with positive and negative affect and stress reactivity in daily life: Findings from a smart phone study. Pers Individ Differ. 2015;87:288-92. doi: 10.1016/j.paid.2015.08.009.

10. Grigaitytė I, Söderberg P. Why does perceived social support protect against somatic symptoms: Investigating the roles of emotional selfefficacy and depressive symptoms? Nord Psychol. 2021;73(3):226-41. doi: 10.1080/19012276.2021.1902845.

11. Yeshua M, Zohar AH, Berkovich L. "Silence! The body is speaking" - a correlational study of personality, perfectionism, and self-compassion as risk and protective factors for psychosomatic symptoms distress. Psychol Health Med. 2019;24(2):229-40. doi: 10.1080/13548506.2018.1546016. [PubMed: 30427205].

12. Ferrari M, Yap K, Scott N, Einstein DA, Ciarrochi J. Self-compassion moderates the perfectionism and depression link in both adolescence and adulthood. PLoS One. 2018;13(2). e0192022. doi: 10.1371/journal.pone.0192022. [PubMed: 29466452]. [PubMed Central: PMC5821438].

13. Jeon H, Lee K, Kwon S. Investigation of the Structural Relationships Between Social Support, Self-Compassion, and Subjective Well-Being in Korean Elite Student Athletes. Psychol Rep. 2016;119(1):39-54. doi: 10.1177/0033294116658226. [PubMed: 27381414].

14. Ling Y, Hu X, Liu C, Huebner E, Wei Y. The Interaction Between Peer Social Support and Stressors Predicts Somatic and Psychological Depressive Symptoms in Chinese Adolescents. Appl Res Qual Life. 2021;17(1):333-49. doi: 10.1007/s11482-020-09883-6.

15. Yu NX, Chan JSM, Ji X, Wan AHY, Ng SM, Yuen LP, et al. Stress and psychosomatic symptoms in Chinese adults with sleep complaints: me- diation effect of self-compassion. Psychol Health Med. 2019;24(2):24152. doi: 10.1080/13548506.2018.1546014. [PubMed: 30422675].

16. Rajabi G, Maghami E. [Self-compassion as mediator between attachment dimensions with mental well-being: a path analysis model]. Health Psychol. 2015;4(4):83-100. Persian.

17. Sherbourne CD, Stewart AL. The MOS social support survey. Soc Sci Med.1991;32(6):705-14. doi:10.1016/0277-9536(91)90150-b.

18. Togari T, Yokoyama Y. Application of the eight-item modified medical outcomes study social support survey in Japan: a national representative cross-sectional study. Qual Life Res. 2016;25(5):1151-8. doi 10.1007/s11136-015-1155-8. [PubMed: 26452364].

19. Neff KD. Erratum to: The Self-Compassion Scale is a Valid and Theoretically Coherent Measure of Self-Compassion. Mindfulness. 2016;7(4):1009. doi: 10.1007/s12671-016-0560-6.

20. Herzog A, Voigt K, Meyer B, Wollburg E, Weinmann N, Langs $\mathrm{G}$, et al. Psychological and interactional characteristics of patients with somatoform disorders: Validation of the Somatic Symptoms Experiences Questionnaire (SSEQ) in a clinical psychosomatic population. J Psychosom Res. 2015;78(6):553-62. doi: 10.1016/j.jpsychores.2015.03.004. [PubMed: 25840951].

21. Yu M, Qiu T, Liu C, Cui Q, Wu H. The mediating role of perceived social support between anxiety symptoms and life satisfaction in pregnant women: a cross-sectional study. Health Qual Life Outcomes. 2020;18(1):223. doi: 10.1186/s12955-020-01479-w. [PubMed: 32650793]. [PubMed Central: PMC7348126].

22. Shahabinejad Z, Zandi S, Azizmohammadi S. [Predictability of University Students' Internet Addiction based on Self-Compassion and Perceived Social Support]. Knowl Res Appl Psychol. 2018;18(4):80-91. Persian.

23. Yaghoubi S, Birashk B, Aghebati A, Ashouri A. Mediating Role of External Shame and Self-compassion in the Relationship Between Peer Victimization and Depression in Adolescents. Iran J Psychiatry Clin Psychol. 2021;27(1):16-31. doi: 10.32598/ijpcp.27.1.3288.1.

24. Terry ML, Leary MR. Self-compassion, self-regulation, and health. Self Identity. 2011;10(3):352-62. doi: 10.1080/15298868.2011.558404. 